\title{
Light-superconducting interference devices
}

\author{
Frans Godschalk and Yuli V. Nazarov \\ Kavli Institute of Nanoscience, Delft University of Technology, P.O. Box 5046, 2600 GA Delft, The Netherlands
}

(Received 28 November 2013; revised manuscript received 14 February 2014; published 3 March 2014)

\begin{abstract}
Recently, we have proposed the half-Josephson laser (HJL): a device that combines lasing with superconducting leads, providing a locking between the optical phase and the superconducting phase difference between the leads. In this work, we propose and investigate two setups derived from a superconducting quantum interference device (SQUID), where two conventional Josephson junctions are replaced by two HJLs. In the first setup, the HJLs share the same resonant mode, while in the second setup two separate resonant modes of the two lasers are coupled optically. We dub the setup "light-superconducting interference device" (LSID). In both setups, we find the operating regimes similar to those of a single HJL. Importantly, the steady lasing field is significantly affected by the magnetic flux penetrating the SQUID loop, with respect to both amplitude and phase. This provides opportunities to tune or even quench the lasing by varying a small magnetic field. For the second setup, we find a parameter range where the evolution equation for the laser fields supports periodic cycles. The fields are thus modulated with the frequency of the cycle resulting in an emission spectrum consisting of a set of discrete modes. From this spectrum, two modes dominate in the limit of strong optical coupling. Therefore, the LSID can be also used to generate such modulated light.
\end{abstract}

DOI: 10.1103/PhysRevB.89.104502

PACS number(s): 42.55.Px, 85.25.Cp, 74.78.Na, 85.25.Dq

\section{INTRODUCTION}

In the past decade there has been a rapidly growing interest in devices that combine semiconductor nanostructures with superconductors. The advantage of this combination lies in the ability of the current-day semiconductor technology to engineer all kinds of devices and nanostructures. When incorporated in a Josephson junction, these determine the transport properties of the junction [1], which, for example, allow manipulation of supercurrents [2], realization of Majorana states [3], and facilitation of superradiant emission of light [4], or become useful for the purposes of quantum manipulation [5].

Recently, we have proposed the so-called half-Josephson laser (HJL) [6]. It consists of a single quantum emitter with two superconducting leads biased at voltage $V$, and an optical cavity with resonant mode at frequency $\approx e V / \hbar$. It emits coherent laser light at a frequency that is precisely $e V / \hbar$, a half of the Josephson frequency, the optical phase of this light being locked with the superconducting phase difference between the leads. A HJL can be viewed as a voltagebiased Josephson junction. Later, after the HJL proposal, we investigated HJLs with multiple emitters, that provide exponentially long coherence times for the emitted light [7], and proposed schemes to reduce noise in the superconducting phase using optical feedback [8].

In this article, we report a study of a HJL application that is built on one of the archetypical devices made of Josephson junctions: the superconducting quantum interference device (SQUID). We consider the dc SQUID setup [9], which is a circuit of two parallel Josephson junctions that supports a supercurrent up to a certain critical value. The whole structure is a superconducting loop, which can be threaded by a magnetic flux. The presence of flux makes the phase drops at the Josephson junctions unequal. As a result, the critical current of the device depends periodically on the flux [10], so that the SQUID can be viewed as a flux-tunable Josephson junction.
The subjects of our study are two SQUID setups where the Josephson junctions are replaced with HJLs. In the simplest case of optically uncoupled HJLs, the effect of the magnetic flux on the superconducting phases, combined with the phase lock of these to the optical phases in the HJLs, will lead [11] to an optical analog of the Aharonov-Bohm [12] effect: the optical interference of the light emitted from the two HJLs in the SQUID depends periodically on the flux through the superconducting loop. In the present study, we will make a step forward by including optical coupling in two ways: (i) the HJLs share a single resonant mode, and (ii) the separate resonant modes of the HJLs are coupled and (partly) hybridized. We will call these setups light-superconducting interference devices (LSIDs).

The paper is organized as follows. In an introductory Sec. II, we explain the essential results and equations for a single HJL. We introduce two SQUID-based setups in Sec. III. These two setups are treated in Secs. IV and V, respectively. The second setup provides a special regime where we find time-dependent periodic lasing solutions. This is investigated in a separate Sec. VI. We conclude in Sec. VII.

\section{INTRODUCTION: THE HALF-JOSEPHSON LASER}

The setup of the LSID is based on individual HJLs that are coupled optically. The dynamics of the optical fields of the LSID is therefore determined by the equations of motion of the single HJLs augmented with interaction terms, as is shown in the following section. With the HJL being a fundamental building block of the LSID setups, we start with repeating the main equations and results for a simple but general model of a multi-emitter HJL. This model was first formulated in Ref. [7]. The results for the model of the single HJL are also useful because many of these are similar to those of the LSID, as is shown later.

The half-Josephson laser can be regarded as a superconductor- $p$ - $n$ diode-superconductor heterostructure 
mounted in an optical resonator. The $p-n$ diode is capable of emitting light by electron-hole recombination [11]. In the model of Ref. [7], the optical resonator mode is driven by a large number of quantum emitters, that form a dipole moment oscillating at about half the Josephson frequency, $\omega_{j} / 2=e V / \hbar$, with $V$ the bias voltage. It is essential that optically active eigenstates of the quantum emitters couple to the two superconducting leads. This coupling then results in a phase lock between the optical phase of the electric field in the resonator mode and the superconducting phase difference between the leads. With increasing field intensity in the mode, the dipole moment saturates, so that steady-state lasing occurs at finite field intensity. In a toy model, the HJL is driven by an ac Josephson current with frequency $\omega_{j}$. The lasing in the HJL occurs as a result of a parametric resonance instability at $\omega_{j} / 2$. Due to this, there are two stable lasing states with optical phases shifted by $\pi$.

Fluctuations in the laser intensity and phase of the HJL originate from quantum noise in the optical mode [13] and spontaneous switchings between eigenstates of the quantum emitters. Such fluctuations can lead to spontaneous switchings between two stable lasing states and result in loss of optical coherence. However, we have shown that the typical switching times can be exponentially long [7]. Therefore, in this work, we consider neither noise nor switching in the devices under consideration.

In Ref. [7], we derived a general model for the HJL applying to an arbitrary set of quantum emitters. The states of these quantum emitters were assumed to couple only weakly to both the optical field and the superconducting leads. This allowed us to express the dipole moment in terms of an expansion in the optical field of the resonant mode and the pair potentials of the superconducting leads. Here, the optical field is represented by the expectation value of the photon annihilation operator, $b \equiv\langle\hat{b}\rangle$. The semiclassical equation of motion of $b$ has the usual form for an oscillator mode that is driven by a dipole moment. It is given by

$$
\dot{b}=-\left(i \omega+\frac{\Gamma}{2}\right) b-i \Omega^{\prime \prime}|b|^{2} b-i A b^{*} e^{i \phi_{\Delta}} .
$$

Here, $\omega$ is the detuning from the frequency of the resonant mode $\omega_{0}, \omega \equiv \omega_{0}-e V / \hbar, \Gamma$ is the decay rate of the mode, and $\phi_{\Delta}$ the superconducting phase difference. The coefficients $\Omega^{\prime \prime}$ and $A$ correspond to the third-order terms in the aforementioned expansion of the dipole moment, where the superconducting potentials are absorbed into $A$. The $\Omega^{\prime \prime}$ term describes the saturation of the dipole moment with increasing photon number in the resonator. The real part of the $A$ term provides the gain that is responsible for driving the resonator mode. Lasing occurs when the gain counters the losses, represented by $\Gamma$. The lowest order term of the expansion of the dipole moment is proportional to $b$ and shifts the resonant frequency of the mode. This shift is already incorporated in the definition of $\omega_{0}$. The second-order terms of the dipole moment expansion are zero, so that no terms occur in Eq. (1) that are quadratic in $b$. This indicates that without the driving, caused by the $A$ term, no photons occupy the resonator. A coherent state of radiation is formed in the resonant mode, with the average photon number being given by $n=|b|^{2}$. The equations are similar to generic equations describing parametric resonance in the presence of a weak nonlinearity [14]. The superconductivity plays the role of an ac drive at double frequency $2 e \mathrm{~V} / \hbar$.

The stationary solutions to Eq. (1) are given by $n=0$ and

$$
\begin{gathered}
n_{ \pm}=\frac{1}{\left|\Omega^{\prime \prime}\right|}\left[ \pm \sqrt{A^{2}-\Gamma^{2} / 4}+\omega\right], \\
\frac{\Gamma}{2} \tan \left(\varphi_{b}-\frac{\phi_{\Delta}}{2}\right)=-A \mp \sqrt{A^{2}-\Gamma^{2} / 4},
\end{gathered}
$$

where we have assumed $\Omega^{\prime \prime}<0$. Here, $\varphi_{b}$ is the optical phase of the field in the mode. The fixed value of the optical phase implies a phase lock to the superconducting phase difference. The solution for the phase is covariant under $\varphi_{b} \rightarrow \varphi_{b}+\pi$, which implies the occurrence of two solutions for each of the $n_{ \pm}$, with opposite field amplitudes.

The existence of stationary solutions is not enough to guarantee the possibility of lasing in the HJL. For this, the stationary solutions must also be stable against small perturbations. Stability analysis of a solution is done by linearizing the equations of motion about this solution. These linearized equations can be solved exactly. If a perturbation always decays back to the stationary solution, the stationary solution is said to be stable. If the perturbation grows, the stationary solution is unstable.

To realize lasing in the HJL, at least one of the solutions $n_{ \pm}$must be real and positive. This condition allows us to distinguish three regimes, depending on the number of physical solutions. (i) Both $n_{ \pm}$are negative [case (i) a] or complex [case (i) b; here $n_{+}=n_{-}^{*}$ ]. The only physical solution to Eq. (3) is at $n=0$. (ii) Only $n_{+}$is real and positive. There are now two physical solutions, of which the one at $n=0$ is unstable against perturbations. This is the regime where we have stable, steady-state lasing with $n_{+}$photons in the mode. To have a large number of photons, it is required that $\left|\Omega^{\prime \prime}\right| \ll \sqrt{A^{2}-\Gamma^{2} / 4}+\omega$. (iii) Both $n_{ \pm}$are real and positive, so that there are three physical solutions. Stability analysis shows that only the solution with $n_{-}$photons in the resonator mode is unstable. Hence this regime is bistable, with both the nonlasing state and the lasing state $\left(n_{+}\right)$stable against perturbations.

In a phase diagram of $2 A / \Gamma$ versus $2 \omega / \Gamma$, regime (i) b borders regimes (i) a and (iii). The boundary is simply defined by $A=\Gamma / 2$. Furthermore, regime (i) a borders (ii) and (ii) borders (iii). Here, the boundaries are respectively given by $\pm|\omega|=\sqrt{A^{2}-\Gamma^{2} / 4}$.

In a steady lasing state a constant current runs through the HJL. Since each photon that escapes the resonator is replenished by an electron-hole pair annihilation, the current is given by the number of photons that escape the cavity, $\Gamma n$, times the electric charge, $I=e \Gamma n$.

\section{SETUPS}

Let us introduce two LSID setups and the corresponding equations of motion for optical fields. The first setup contains two HJLs sharing a single cavity, which are embedded in the arms of a superconducting loop. This is similar to the design of a dc SQUID. A magnetic flux $\Phi$ threads the loop of the SQUID structure, thus relating the superconducting phase differences 
across the Josephson junctions, defined by $\phi_{\Delta}^{(j)}$ for $j=1,2$, so that $\phi_{\Delta}^{(1)}-\phi_{\Delta}^{(2)}=2 \pi \Phi / \Phi_{0}$. Here $\Phi_{0}=\pi \hbar / e$ is the quantum of magnetic flux. From now, we refer to this setup as "singlemode LSID."

The description of the single-mode LSID is based on the phenomenological model that was introduced with Eq. (1) for a single HJL. We account for two HJLs by splitting the term proportional to $b^{*}$. Each part comes with its own coefficient, $A_{j}, j=1,2$, and the corresponding superconducting phase difference, $\phi_{\Delta}^{(j)}$. Redefining the optical phase of $b, \varphi_{b} \rightarrow \varphi_{b}+$ $\phi_{\Delta}^{(1)} / 2$, we arrive at

$$
\begin{aligned}
\dot{b}= & -\left(i \omega+\frac{\Gamma}{2}\right) b-i \Omega^{\prime \prime}|b|^{2} b \\
& -i A_{1} b^{*}-i A_{2} b^{*} e^{-2 i \pi \Phi / \Phi_{0}} .
\end{aligned}
$$

Here, $\omega, \Gamma$, and $\Omega^{\prime \prime}$ are the same as in Eq. (1), while $A_{1}$ and $A_{2}$ are equivalent to $A$ in the model of a single HJL. Also here, without loss of generality, we assume from now on $\Omega^{\prime \prime}<0$. In the case of similar HJLs in the arms of the SQUID, $A_{1} \simeq A_{2}$. We note that the equation of motion for a single $\mathrm{HJL}$ is obtained by setting $\Phi=0$.

The second setup is similar to the first one, with the exception that there are now two resonant modes, each associated with a HJL. The modes are coupled optically. For instance, this can be realized if each HJL is mounted in a separate optical cavity, the cavities being connected with a fiber. Also here, a flux $\Phi$ threads the loop. This device will be referred to as "two-mode LSID."

We model this setup using two copies of the equations of motion for a single-mode HJL, Eq. (1), and by augmenting those with a coupling term [15]. Assuming $b_{j}$ to be the optical field in the modes labeled with $j=1,2$, we arrive at

$$
\begin{aligned}
\dot{b}_{1}= & -\left(i \omega_{1}+\frac{\Gamma_{1}}{2}\right) b_{1}-i \Omega_{1}^{\prime \prime}\left|b_{1}\right|^{2} b_{1} \\
& -i A_{1} b_{1}^{*}-i g b_{2} e^{-i \pi \Phi / \Phi_{0}}, \\
\dot{b}_{2}= & -\left(i \omega_{2}+\frac{\Gamma_{2}}{2}\right) b_{2}-i \Omega_{2}^{\prime \prime}\left|b_{2}\right|^{2} b_{2} \\
& -i A_{2} b_{2}^{*}-i g b_{1} e^{i \pi \Phi / \Phi_{0}} .
\end{aligned}
$$

Here, $\omega_{j}$ is the detuning of each mode and $\Gamma_{j}$ the decay rate. The coefficients $\Omega_{j}^{\prime \prime}$ and $A_{j}$ are coefficients of the expansion of the dipole moments. Like for the model of the single mode, we redefined here the optical phases: $\phi_{b}^{(j)} \rightarrow \phi_{b}^{(j)}+\phi_{\Delta}^{(j)} / 2$. The coupling between the modes is proportional to coupling strength $g$, which we take real, without loss of generality.

It is worth noting that, compared to the first setup, the second setup has more output quantities: one can separately measure intensity and optical phase of the light emitted from each mode. Schematics of both setups are shown in Fig. 1.

\section{SINGLE-MODE LSID}

Let us now analyze the model of the single mode LSID. The stationary solutions to Eq. (3) yield the stationary number of photons in the resonator mode, $n=|b|^{2}$, and the optical (a)

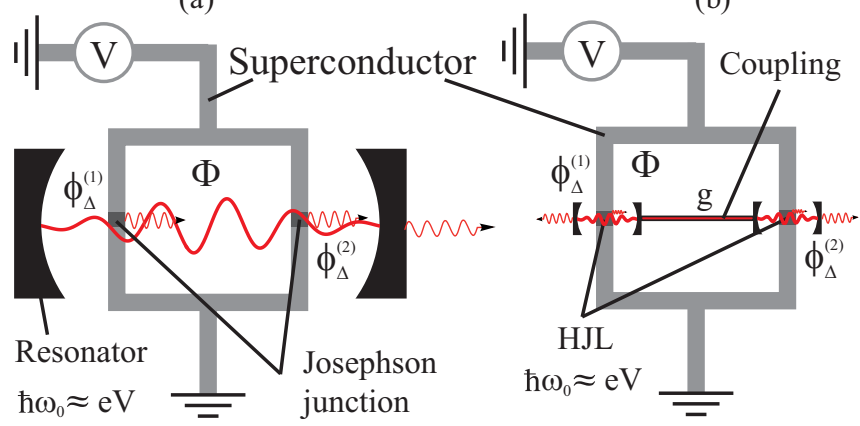

FIG. 1. (Color online) Setups. (a) The single-mode LSID. Two HJLs, sharing the same optical cavity, are embedded in a superconducting loop. The resonant frequency is $\omega_{0} \approx e V / \hbar$. (b) The dual-mode LSID: a superconducting loop containing a HJL in each arm. The HJLs are embedded in separate cavities with resonant-mode frequencies $\omega_{1,2} \approx e V / \hbar$. The optical coupling between the resonant modes is characterized with a parameter $g$, such that the splitting of the frequencies of the hybridized modes equals $2 g$.

phase. They are given by

$$
\begin{aligned}
\left|\Omega^{\prime \prime}\right| n_{ \pm} & = \pm \sqrt{A_{1}^{2}+A_{2}^{2}+2 A_{1} A_{2} \cos \left(2 \pi \frac{\Phi}{\Phi_{0}}\right)-\frac{\Gamma^{2}}{4}}+\omega, \\
\tan 2 \varphi_{b}^{ \pm} & =\frac{\frac{\Gamma}{2}\left[A_{1}+A_{2} \cos \left(2 \pi \frac{\Phi}{\Phi_{0}}\right)\right] \mp W A_{2} \sin \left(2 \pi \frac{\Phi}{\Phi_{0}}\right)}{ \pm W\left[A_{1}+A_{2} \cos \left(2 \pi \frac{\Phi}{\Phi_{0}}\right)\right]+\frac{\Gamma}{2} A_{2} \sin \left(2 \pi \frac{\Phi}{\Phi_{0}}\right)},
\end{aligned}
$$

with $W=\omega-\left|\Omega^{\prime \prime}\right| n_{ \pm}$. Besides, $n=0$ is also a stationary solution. The expression for $\varphi_{b}$ implies that a stationary state with photon number $n_{ \pm}$can occur with two phases, differing by $\pi$. Furthermore, the physical solutions correspond to real and positive $n_{ \pm}$. As a minimal requirement for lasing, we need $\left|A_{1}+A_{2}\right|>\Gamma / 2$. From now on, we assume this to be the case. It is essential to note that the $n_{ \pm}$depend on the magnetic flux. In particular, the threshold values of $\omega$ at which $n_{ \pm}=0$ depend periodically on $\Phi: \omega_{\mathrm{thr}}^{ \pm}(\Phi)$. The sensitivity to flux is highest when $\left|A_{1}-A_{2}\right|<\Gamma / 2$. In this case, there is a value of $\Phi$, where the expression in the square root becomes zero, so that $n_{-}=n_{+}=\omega /\left|\Omega^{\prime \prime}\right|$.

The single-mode LSID can operate in three different regimes [7]. The definition of these regimes is exactly the same as that of those of the single HJL, while the boundaries separating the regimes are different and the dimensionality of the phase diagram is higher, involving the extra parameters $A_{2}$ and $\Phi$.

It is possible to switch between the regimes by changing parameters. For instance, for a single HJL (equivalent to setting $\Phi=0$ for the single-mode LSID) one can switch from regime (i) to (ii) and from (ii) to (iii) by sweeping the voltage and thus the detuning $\omega$. With the single-mode LSID, new possibilities arise to switch between the regimes. A very interesting one is a switch between regimes (i) and (ii), a nonlasing and a lasing regime, by changing the flux only. This can happen in two ways. First, we can choose $\omega$ such 


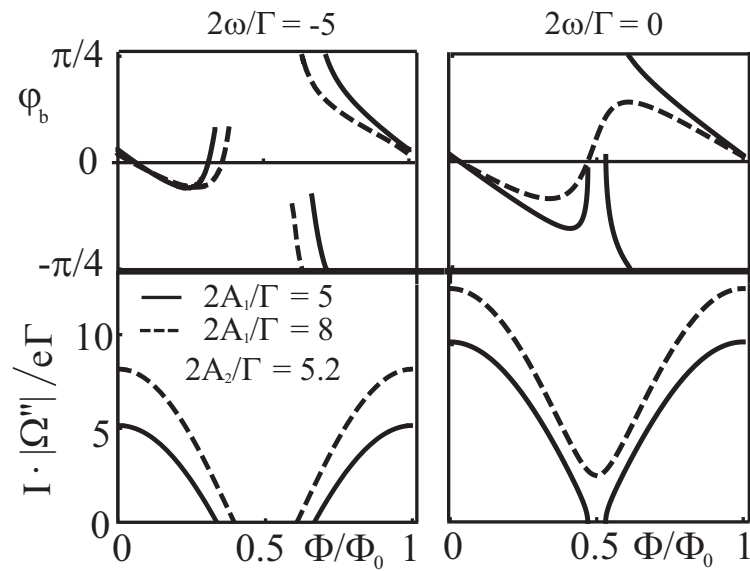

FIG. 2. Flux dependence of the current and optical phase, and regime switching for the single-mode LSID. In the lower panels, the current through the device is plotted as a function of the flux, $\Phi$. The current is proportional to the $n_{+}$given by Eq. (5). In the upper panels, the corresponding optical phases are plotted. For the solid (dashed) lines $\left|A_{1}-A_{2}\right|<\Gamma / 2\left(\left|A_{1}-A_{2}\right|>\Gamma / 2\right)$. For the leftmost panels, the detuning is chosen such that the HJL undergoes a transition between the regimes (ii) and (i) a described in the main text. This occurs near the half of a flux quantum. This happens for both cases corresponding to the dashed and the solid lines. For the rightmost panels, no transition occurs for the dashed line, while a transition from regime (ii) to (i) $b$ occurs for the solid line, in the vicinity of half of a flux quantum.

that it is crossed by $\omega_{\mathrm{thr}}^{+}\left(\Phi_{\mathrm{thr}}^{+,(i)}\right)$ at the threshold value of the flux, $\Phi_{\mathrm{thr}}^{+,(i)}$. This is a transition between regimes (i) a and (ii). It corresponds to a second-order phase transition, where the derivative of $n$ to $\Phi$ is finite when the threshold is crossed. The second way occurs when $\left|A_{1}-A_{2}\right|<\Gamma / 2$ and $\omega=0$. Here a second-order phase transition between regimes (i) $b$ and (ii) occurs at the two threshold values of $\Phi$. At this phase transition we find $n_{-}=n_{+}=0$, while the derivative of $n$ to $\Phi$ is infinite. These cases are shown in Fig. 2, where the current through the device and the optical phase are plotted as a function of flux. Hence, with these phase transitions it is possible to switch a single-mode LSID on and off using a magnetic field only.

There is also a parameter regime where the single-mode LSID displays hysteretic behavior upon a flux sweep. This regime occurs when $\left|A_{1}-A_{2}\right|<\Gamma / 2$ and $\omega$ is chosen such that $\omega_{\mathrm{thr}}^{-}\left(\Phi_{\mathrm{thr}}^{-,(i)}\right)=\omega$ (the "threshold" of the unstable solution), at the threshold value of the flux, $\Phi_{\text {thr }}^{-,(i)}$. If we start at $\Phi=0$, the HJL is in regime (ii). Upon increasing $\Phi$ adiabatically, a transition to the bistable regime (iii) takes place when the threshold $\Phi_{\mathrm{thr}}^{-,(1)}$ is crossed. The single-mode LSID remains in the steady lasing state. At a critical value of $\Phi$ we encounter a transition to the nonlasing regime (i) $b$. This is a first-order phase transition, where the single-mode LSID turns off. When we decrease $\Phi$, the transition proceeds in opposite direction, from regime (i) b to (iii). Since the nonlasing state is stable in this regime, the HJL remains off. Crossing $\Phi_{\text {thr }}^{-,(1)}$ another time, we encounter a first-order transition to the original lasing regime (ii). The hysteresis in the HJL is shown in Fig. 3, where

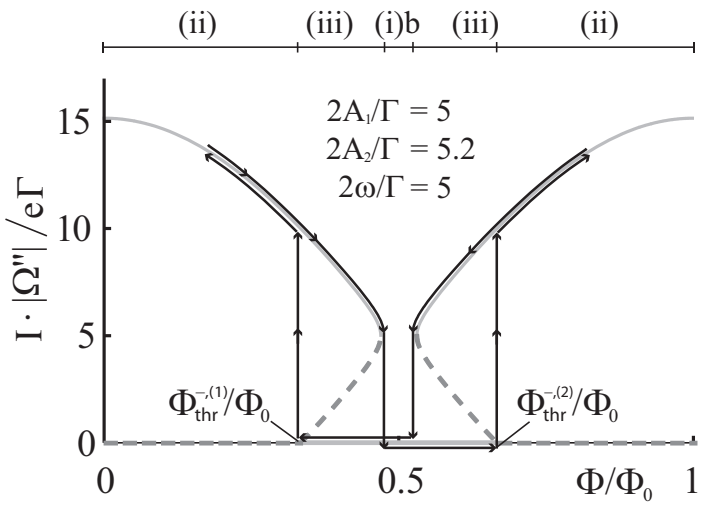

FIG. 3. The hysteresis in the single-mode LSID. The bistable regime (iii) supports hysteretic behavior in the HJL. The gray, solid (dashed) curve represents the current as calculated from the stable (unstable) solution of Eq. (5). The solid (dashed) line at $I=0$ indicates that the nonlasing solution is stable (unstable). The solid black lines represent a flux sweep, with the direction indicated by the arrows. These lines are slightly shifted for clarity. The regimes (i) b, (ii), and (iii), indicated above the plot, and the threshold flux values, $\Phi_{\mathrm{thr}}^{-,(i)}$, are explained in the main text.

the sweep occurs over a wider range of $\Phi$, which also includes a second threshold, $\Phi_{\mathrm{thr}}^{-,(2)}$.

To conclude this section, we have described a single-mode LSID, where two HJLs sharing the same resonant mode are incorporated in a superconducting loop. We find the singlemode LSID to be flux tunable. Importantly, in some parameter regimes the lasing can even be switched on and off solely using the small magnetic fields. Additionally, a parameter regime exists where there is a hysteresis with respect to a flux sweep.

\section{TWO-MODE LSID}

In this section we analyze the model, Eq. (4), of the two-mode LSID, where a superconducting loop contains a HJL in each arm of the loop, while the resonant modes are coupled optically. The relative complexity of this model prohibits us from doing a full analytical study. Instead, we investigate the weak- and the strong-coupling limits using perturbative methods. Then we study analytically the equations for a specific, symmetric choice of parameters, assuming no particular coupling strength. For a particular parameter range of the latter case, we also perform a numerical study, in Sec. VI, where we find time-dependent solutions to Eq. (4).

\section{A. Weak-coupling limit}

Let us first study the weak-coupling limit, $g \ll A_{i}$, of the two-mode LSID. In this limit, the two HJLs in the device only slightly perturb each other. The perturbation depends on the flux, $\Phi$. As a result, the current through the device displays small oscillations upon changing flux.

We calculate the flux-dependent change in the optical fields of the modes in the weak-coupling limit. The stationary lasing states of the uncoupled HJLs, given in Eq. (2), are taken to 
be $n_{j}^{0}=\left|b_{j}^{0}\right|^{2}$ and $\varphi_{b_{j}}^{0}$, with the index $j=1,2$ labeling the HJLs. For clarity, we make an extra assumption $\Gamma_{j} \ll A_{j}$ and expand Eq. (2) about $\Gamma_{j}=0$. In this limit, $\left(x_{j}^{0}, y_{j}^{0}\right)=$ $\left(\sqrt{\left(A_{j}+\omega_{j}\right) /\left|\Omega_{j}^{\prime \prime}\right|}, 0\right)$. The optical coupling in Eq. (4) and the $\Gamma_{j}$ perturb the optical fields as $b_{j}=b_{j}^{0}+\delta b_{j} \equiv x_{j}^{0}+i y_{j}^{0}+$ $\delta x_{j}+i \delta y_{j}$, with $x_{j}^{0}, y_{j}^{0}, \delta x_{j}, \delta y_{j}$ being real. We calculate the linear variations owing to $\Gamma_{j}$ and $g$, which yields

$$
\begin{aligned}
\delta^{\Gamma} x_{j} & =0, \\
\delta^{\Gamma} y_{j} & =-x_{j}^{0} \frac{\Gamma_{j}}{2 A_{j}}, \\
\delta^{g} x_{j} & =x_{k}^{0} \frac{g}{2\left(A_{j}+\omega_{j}\right)} \cos \left[\pi \Phi / \Phi_{0}\right], \\
\delta^{g} y_{j} & =x_{k}^{0}(-1)^{j} \frac{g}{2 A_{j}} \sin \left[\pi \Phi / \Phi_{0}\right],
\end{aligned}
$$

where $j, k=1,2$ and $j \neq k$.

As a result of the perturbative interaction, the current through the $\mathrm{HJL}$ and the optical phase change. The total current through the device becomes $I \simeq I_{0}+\delta I$, with $I_{0}=e \sum_{j} \Gamma_{j} n_{j}^{0}$ and $\delta I=2 e \sum \Gamma_{i}\left(x_{i}^{0} \delta x_{i}+y_{i}^{0} \delta y_{i}\right)$. Here $I_{0} \simeq$ $\sqrt{n_{0}} \delta I$. The variations owing to $\Gamma_{j}$ yield a small constant reduction of the total current in second order, while the ones owing to the optical coupling yield, in first order, a small fluxdependent change of the current. The perturbation to the optical phase is given by $\delta \varphi_{b_{j}}=\cos ^{2}\left[\pi \Phi / \Phi_{0}\right]\left(\delta y_{j}^{0}-\tan \varphi_{b_{j}}^{0} \delta x_{j}^{0}\right) / x_{j}^{0}$. Up to first order, we find

$$
\begin{aligned}
\delta I & =e g\left[\frac{\Gamma_{1}}{\left|\Omega_{1}^{\prime \prime}\right|} \sqrt{\frac{n_{2}^{0}}{n_{1}^{0}}}+\frac{\Gamma_{2}}{\left|\Omega_{2}^{\prime \prime}\right|} \sqrt{\frac{n_{1}^{0}}{n_{2}^{0}}}\right] \cos \left[\pi \Phi / \Phi_{0}\right], \\
\delta \varphi_{b_{j}} & =\frac{\cos ^{2}\left[\pi \Phi / \Phi_{0}\right]}{2 A_{j}}\left[-\Gamma_{j}+(-1)^{j} g \sqrt{\frac{n_{k}^{0}}{n_{j}^{0}}} \sin \left[\pi \Phi / \Phi_{0}\right]\right],
\end{aligned}
$$

where $k \neq j$ and $n_{j}^{0}=\left(x_{j}^{0}\right)^{2}=\left(A_{j}+\omega_{j}\right) /\left|\Omega_{j}^{\prime \prime}\right|$. The phase variation can be written as a sum of simple harmonic functions, with arguments $m \pi \Phi / \Phi_{0}$, for $m=1,2,3$.

\section{B. Strong-coupling limit}

We proceed with the strong-coupling limit of the two-mode LSID assuming $g \gg A_{i}, \Gamma_{i}$. In this limit, the modes of the HJLs are essentially hybridized. The frequencies of the hybridized modes are shifted by $\pm g$. We show that each of these hybridized modes is excited separately in separate ranges of detuning. In these ranges, the two-mode LSID works similar to a single-mode LSID.

A perturbative treatment of Eq. (4) requires tuning to one of the two hybridized modes, $\omega_{i} \simeq \pm g$. With this, the lowest order stationary solution reads $\omega_{2} b_{2}^{(0)}=-g b_{1} e^{i \pi \Phi / \Phi_{0}}$. Then, up to first order we have $\pm \omega_{2} b_{2}=\left[ \pm \omega_{2}+i \Gamma_{2}-\left|\Omega_{2}^{\prime \prime}\right| n_{2}^{(0)}\right] b_{2}^{(0)}-$ $A_{2}\left[b_{2}^{(0)}\right]^{*}$. Inserting these results in the expression for $b_{1}$ yields the equation for a stationary single-mode LSID:

$$
\begin{aligned}
{[ \pm} & \left.i\left(\omega_{1}-\frac{g^{2}}{\omega_{2}^{2}} \omega_{2}\right)+\frac{\Gamma_{1}}{2}+\frac{\Gamma_{2}}{2} \frac{g^{2}}{\omega_{2}^{2}}\right] b_{1} \\
= & -i\left(\Omega_{1}^{\prime \prime}+\frac{g^{4}}{\omega_{2}^{4}} \Omega_{2}^{\prime \prime}\right) n_{1} b_{1} \\
& -i\left(A_{1}+\frac{g^{2}}{\omega_{2}^{2}} A_{2} e^{-2 i \pi \Phi / \Phi_{0}}\right) b_{1}^{*} .
\end{aligned}
$$

The approximation leading to this equation is valid for a limited range of detunings, $\left|\omega_{1}-\left(g^{2} / \omega_{2}\right)\right| \lesssim\left|A_{j}\right|$.

\section{Symmetric equations}

The limits studied so far give a rather narrow perspective of the two-mode LSID: in the weak-coupling limit it is described as two largely independent HJLs, while in the strong-coupling limit it essentially becomes a single-mode LSID, at least for a narrow interval of detuning. To learn more about the device, let us assume the HJLs to be identical. With this, it is possible to analytically calculate stationary solutions to Eq. (4). Small deviations from this assumption of symmetry can in principal be treated perturbatively. Doing so, we have not found any qualitative differences from the symmetric case. Hence, we describe all essentials of the two-mode LSID for the case when the arms of the superconducting loop contain equal HJLs.

Before presenting the stationary solutions, we first reduce the parameter space of Eq. (4) by rescaling various quantities to dimensionless form,

$$
\begin{aligned}
\tilde{b}_{j} & \equiv \sqrt{\frac{\left|\Omega_{j}^{\prime \prime}\right|}{A_{j}}} b_{j}, \quad \gamma_{j} \equiv \Gamma_{j} /\left(2 A_{j}\right), \\
G_{j k} & \equiv \frac{g}{A_{j}} \sqrt{\frac{\left|\Omega_{j}^{\prime \prime}\right|}{\left|\Omega_{k}^{\prime \prime}\right|} \frac{A_{k}}{A_{j}}}, \quad \tilde{\omega}_{j} \equiv \omega_{j} / A_{j},
\end{aligned}
$$

and measuring time in units of $\left(A_{1} A_{2}\right)^{-1 / 2}$. With this, the equations of motion become

$$
\begin{aligned}
\sqrt{\frac{A_{2}}{A_{1}}} \dot{\tilde{b}}_{1}= & -\left(i \tilde{\omega}_{1}+\gamma_{1}\right) \tilde{b}_{1}+i\left|\tilde{b}_{1}\right|^{2} \tilde{b}_{1} \\
& -i \tilde{b}_{1}^{*}-i G_{12} \tilde{b}_{2} e^{-i \pi \Phi / \Phi_{0}}, \\
\sqrt{\frac{A_{1}}{A_{2}}} \dot{\tilde{b}}_{2}= & -\left(i \tilde{\omega}_{2}+\gamma_{2}\right) \tilde{b}_{2}+i\left|\tilde{b}_{2}\right|^{2} \tilde{b}_{2} \\
& -i \tilde{b}_{2}^{*}-i G_{21} \tilde{b}_{1} e^{i \pi \Phi / \Phi_{0}} .
\end{aligned}
$$

The assumption of symmetry implies $\tilde{\omega}_{1}=\tilde{\omega}_{2}=\tilde{\omega}, \gamma_{1}=$ $\gamma_{2} \equiv \gamma$, and $G \equiv G_{12}=G_{21}$ (we note that this does not imply $A_{1}=A_{2}$ ). For this choice, the stationary solutions of the equations of motion are invariant under exchange of the resonators $(1 \leftrightarrow 2)$ and reversing the magnetic field $\Phi \rightarrow-\Phi$. Because of this $\tilde{n} \equiv \tilde{n}_{1}=\tilde{n}_{2}$, with $\tilde{n}_{j}=\left|\tilde{b}_{j}\right|^{2}$, while $\varphi_{b_{1}} \neq \varphi_{b_{2}}$.

We have found five stationary solutions, either stable or unstable, to Eqs. (10) in the symmetric case, for photon number and optical phase. This includes $n=0$. For brevity, we give 
the expression of the optical phases only in the limit $\gamma \rightarrow 0$ :

$$
\begin{aligned}
& \tilde{n}_{\alpha}^{ \pm}= \pm \sqrt{1+G^{2}-\gamma^{2}+2 G \sqrt{\cos ^{2}\left(\pi \frac{\Phi}{\Phi_{0}}\right)-\gamma^{2}}}+\tilde{\omega}, \\
& 2 \varphi_{b, \alpha}^{(1)}+\frac{\pi \pm \pi}{2}=\arctan \left[\frac{-G \sin \left(\pi \frac{\Phi}{\Phi_{0}}\right)}{1+G \cos \left(\pi \frac{\Phi}{\Phi_{0}}\right)}\right] \equiv \varphi_{G}^{+}, \\
& \text {with } \varphi_{b, \alpha}^{(1)}=-\varphi_{b, \alpha}^{(2)} \text {, } \\
& \tilde{n}_{\beta}^{ \pm}= \pm \sqrt{1+G^{2}-\gamma^{2}-2 G \sqrt{\cos ^{2}\left(\pi \frac{\Phi}{\Phi_{0}}\right)-\gamma^{2}}}+\tilde{\omega} \\
& 2 \varphi_{b, \beta}^{(1)} \mp \frac{\pi}{2}=\arctan \left[\frac{G \sin \left(\pi \frac{\Phi}{\Phi_{0}}\right)}{1-G \cos \left(\pi \frac{\Phi}{\Phi_{0}}\right)}\right] \equiv \varphi_{G}^{-}, \\
& \text {with } \varphi_{b, \beta}^{(1)}=-\varphi_{b, \beta}^{(2)}-\frac{\pi}{2} \text {. }
\end{aligned}
$$

Let us make several remarks. First, the solutions are invariant under a change of both optical phases with $\pi$. This is equivalent to the invariance of Eq. (10) under a sign change of both $\tilde{b}_{1}$ and $\tilde{b}_{2}$. Second, the solutions are periodic in flux, with the flux period of $2 \Phi_{0}$. This period is however only visible in the dependence of the optical phases on the flux, that can be probed by measuring the light interference. In contrast, the current through the device is only sensitive to the photon number, which has a flux period of $\Phi_{0}$. Finally, for $\gamma \neq 0$ there is a region of flux values, defined by $\gamma>\left|\cos \left[\pi \Phi / \Phi_{0}\right]\right|$, where the $\tilde{n}_{\alpha, \beta}^{ \pm}$are complex valued, so that the only physical solution is at $\tilde{n}=0$. This regime is similar to the regime (i) b that was discussed in context of the single-mode LSID in Sec. IV.

Figure 4 presents the plots of the solutions of Eqs. (11) and (12). The solutions $n_{\alpha, \beta}^{ \pm}$are shown in Fig. 4(a) (plot in the center). In this panel, we can distinguish the various regimes that occur in this device; those are similar to the ones introduced for the single-mode LSID in Sec. IV. In regime

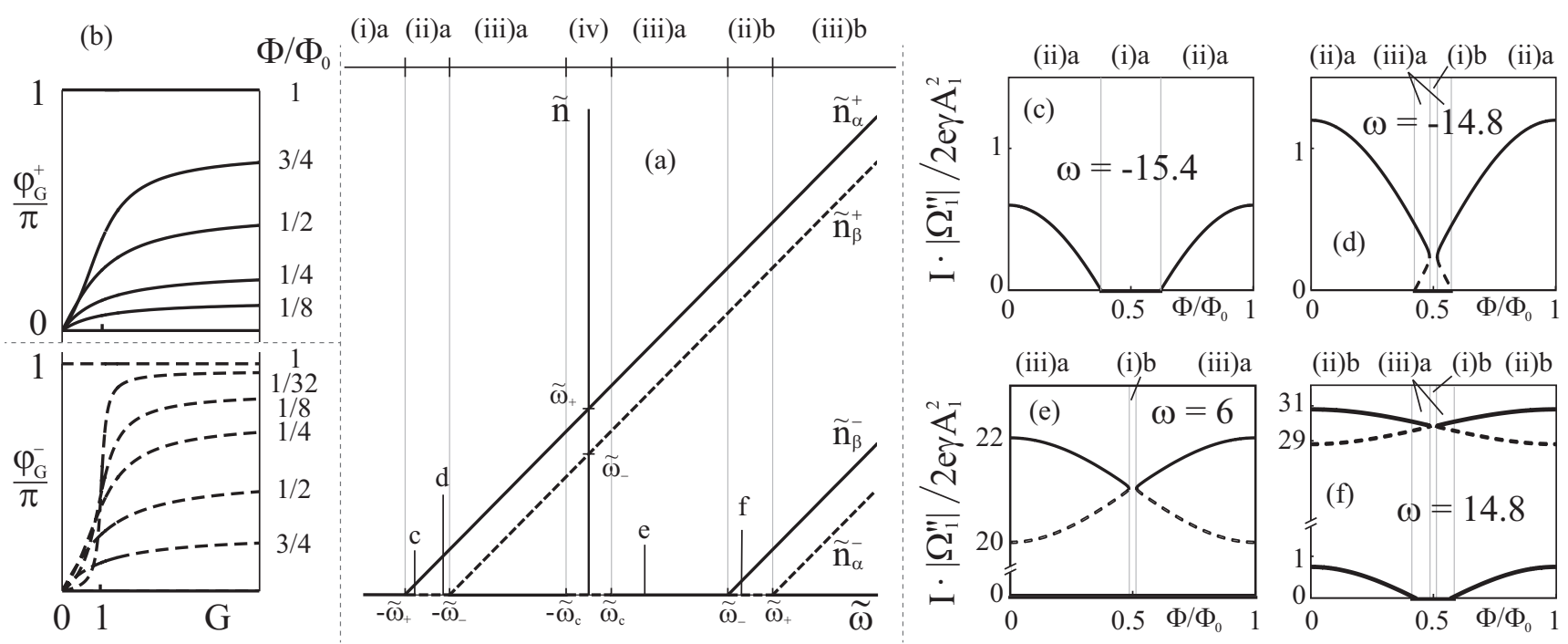

FIG. 4. The lasing in the two-mode LSID. (a) The four solutions of $n$ as given by Eqs. (11) and (12), as a function of detuning. This interval of $\tilde{\omega}$ corresponds to various regimes described in the main text. The regimes are indicated by labels above the plot. The values of detuning labeled with c-f correspond to the panels (c)-(f) at $\Phi=\Phi_{0} / 4$. In the strong-coupling limit, the solid lines labeled with $\tilde{n}_{\alpha}^{+}$and $\tilde{n}_{\beta}^{-}$are the stable stationary solutions, while the dashed lines labeled with $\tilde{n}_{\beta}^{+}$and $\tilde{n}_{\alpha}^{-}$are the unstable ones. We have defined $\tilde{\omega}_{ \pm}^{2} \equiv 1+G^{2}-\gamma^{2} \pm 2 G \sqrt{\cos ^{2}\left[\pi \Phi / \Phi_{0}\right]-\gamma^{2}}$. In the limit of $G \gg 1$, the critical value of the detuning for regime (iv) is given by $\tilde{\omega}_{c}=\sqrt{\sin ^{2}\left[\pi \Phi / \Phi_{0}\right]-\gamma^{2}}$ (Sec. VI). (b) The optical phases as a function of $G$ for several values of the flux, as explained in the main text. The solid (dashed) curves correspond to the solid (dashed) curves in (a), for $0<\Phi \leqslant \Phi_{0}$. (c)-(f) The stationary current (thick solid curves) as a function of flux, for several values of the detuning. The dashed curves correspond to the current at the unstable stationary solutions. Panels (d)-(f) contain bistable regimes where two values of the stationary current are possible. With changing flux, switches between various regimes, indicated above the panels, occur. These panels correspond to the strong-coupling limit, with $G=15$. Furthermore, $\gamma=0.05$ and $\left|\Omega_{1}^{\prime \prime}\right| A_{2}^{2}=\left|\Omega_{2}^{\prime \prime}\right| A_{1}^{2}$. 
(i) a no lasing occurs in the two-mode LSID. Regime (i) b is not shown in the plot, while it was mentioned in the previous paragraph. The lasing occurs in the regime (ii). There is a single stable lasing solution in (ii) a and there are two stable lasing solutions in (ii) b. The latter also involves an unstable lasing solution. Regime (iii) a is bistable while (iii) b is tristable. These regimes also contain one and two unstable solutions, respectively. In both cases, the nonlasing solution is stable. Finally, in the vicinity of $\tilde{\omega}=0$ there is a new regime (iv). This regime contains time-dependent solutions (limit cycles) and will be the topic of investigation in Sec. VI.

We see that steady-state lasing occurs in regime (ii), in two small windows of the detuning, those being in the vicinity of $\tilde{\omega}= \pm G$. Hence, indeed as expected, we find two lasing modes at a frequency shifted by the coupling constant $\simeq G$ and a frequency splitting of $\simeq 2 G$.

The stability of the solutions found depends on the coupling strength. For $G \gg 1$, the solid lines $\left(\tilde{n}_{\alpha}^{+}\right.$and $\left.\tilde{n}_{\beta}^{-}\right)$in Fig. 4(a) represent the stable solutions. The dashed lines represent the unstable ones. In the limit $G \ll 1$ we find that $\tilde{n}_{\beta}^{+}$is stable instead of unstable, while $\tilde{n}_{\beta}^{-}$is unstable instead of stable. This is expected in the regime where the two HJLs in the arms of the superconducting loop are only coupled weakly. Here, both HJLs should lase in a regime of detuning about $\tilde{\omega}=0$. The stable solutions merge at $G \rightarrow 0$, as do the unstable ones.

The dependence of the optical phases on the flux is shown in Fig. 4(b). Instead of showing the value of each solution of the phase separately, we have plotted $\varphi_{G}^{ \pm}$. It is sufficient to plot in a flux interval from zero to $\Phi_{0}$. Indeed, $\varphi_{G}^{ \pm}$for $0<\Phi \leqslant \Phi_{0}$ is the same as $\varphi_{G}^{\mp}$ for $\Phi_{0}<\Phi \leqslant 2 \Phi_{0}$. At $G=0$ the phase $\varphi_{G}^{ \pm}$is either zero or $\pi$ while for $G \rightarrow \infty, \tan \varphi_{G}^{ \pm}= \pm \tan \left[\pi \Phi / \Phi_{0}\right]$.

Finally, Figs. 4(c)-4(f) show the (possible) stationary currents as a function of the flux in the strong-coupling limit. Similarly to the single-mode LSID, the flux can be used to change the operating regime of the device. Panels (c) and (d) correspond to the same regimes as the left and right panels (for the latter only the solid line) of Fig. 2, respectively. Indeed, a flux sweep in the parameter regime of panel (d) would show hysteresis, similar to what is shown in Fig. 3 in Sec. IV. In panel (e) a regime change between the bistable regime (iii) a and the nonlasing regime (i) $b$ occurs. If in the lasing state, a flux sweep across the point $\Phi_{0} / 2$ extinguishes the lasing without recovering. Panel (f) shows transitions between regimes (ii) b, (iii) a, and (i) b.

To conclude this section, we have studied the two-mode LSID. In the weak-coupling limit, the effect of the flux is small periodic modulations at the background of the current for two uncoupled HJLs. In the strong-coupling limit, there are intervals of the detuning where the device operates like the single-mode LSID while the overall picture is more complex. In the next section, we concentrate on a nontrivial feature that is unique for the two-mode LSID.

\section{PERIODIC LASING CYCLES}

In the previous sections, we have studied the stationary states of the LSID. As noted, the two-mode LSID displays a regime with time-dependent steady solutions, or "limit cycles." These are the topic of this section. First, we give a theoretical background to this phenomenon. We do stability analysis to find the parameter ranges where this interesting regime takes place, and identify the corresponding dynamics of the LSID. Then, we use a perturbative analysis in the limit of strong coupling $G \gg 1$ to estimate the key properties of the limit cycles. We find that in this limit, the emission predominantly occurs at two frequencies separated from $e V / \hbar$ by $\pm g$. We refer to this as dual-mode lasing.

After this, we present the results based on the numerical integration of the differential equation and compare these with the theoretical estimates.

\section{A. Stability}

We study the stability of the nonlasing solution, $\tilde{b}_{j}=$ 0 , in the vicinity of $\tilde{\omega}=0$. As in the previous section, we assume equal parameters $\tilde{\omega} \equiv \tilde{\omega}_{1,2}, \quad \gamma \equiv \gamma_{1,2}, G_{12}=$ $G_{21}$. In addition, we assume $A_{1}=A_{2}$. The eigenvalues of the linearized equations of motion in the vicinity $\tilde{b}_{j}=0$ read

$$
\lambda \equiv \gamma \pm \sqrt{1-G^{2}-\tilde{\omega}^{2} \pm 2 G \sqrt{\tilde{\omega}^{2}-\sin ^{2}\left[\pi \Phi / \Phi_{0}\right]}},
$$

for all four possible combinations of the " \pm "s. From this, we can resolve the various regimes defined in Sec. V. For instance, the solution at $n=0$ is stable when the real parts of all $\lambda$ are positive. In the lasing regime (ii) all $\lambda$ are real, yet three are positive and one is negative, thus indicating a saddle point instability. In regime (iv), the nonlasing solution is also unstable, but here the corresponding eigenvalues are complex instead of real, while the real part of two eigenvalues is negative. This regime can only occur if $\tilde{\omega}^{2}<\sin ^{2}\left[\pi \Phi / \Phi_{0}\right]$. In the remainder of this section we will always assume $G \gg 1$, so that regimes (ii) and (iv) are clearly separated from each other. Then $\lambda$ is approximated as

$$
\lambda \simeq \gamma \pm \sqrt{\sin ^{2}\left[\pi \Phi / \Phi_{0}\right]-\tilde{\omega}^{2}} \pm i\left[G+\frac{\tilde{\omega}^{2}-1}{2 G}\right],
$$

again for all four possible choices of the " \pm "s. Therefore, in this limit, the threshold for regime (iv) is defined by $\gamma^{2}+$ $\tilde{\omega}^{2}=\sin ^{2}\left[\pi \Phi / \Phi_{0}\right]$. Crossing this threshold corresponds to a transition from regime (iii) a to regime (iv).

To understand the implications of the transition to regime (iv), let us first consider briefly the dynamics of the two-mode LSID in regime (iii) a. We discuss it in terms used in Sec. IV of Ref. [7]. We assume that the LSID is not in a stationary state. Then the evolution of the state of the device is governed by Eq. (10). The optical fields, $\tilde{b}_{j}$, can be decomposed into real and imaginary parts, $\tilde{b}_{j}=x_{j}+i y_{j}$. Using these, we can construct a four-dimensional coordinate space where each point, $\left(x_{1}, y_{1}, x_{2}, y_{2}\right)$, represents a state of the two-mode LSID. We can map the state evolution to the motion in the coordinate space of a "particle" which is driven by a "force field." Given some initial condition, the particle will evolve along a trajectory defined by the force field, to a stable stationary point or "attractor." The set of initial conditions from which the particle flows to one specific attractor is the domain of attraction of that attractor.

In contrast to the attractors, some stationary points are unstable saddle points. Generally, when close to a saddle point, the particle will be repelled by it. There are however 
trajectories that lead the particle to the saddle point without it being repelled. These trajectories form the stable direction of the saddle point and form a separatrix of Eq. (10). In the cases of regimes (ii) a and (iii) a of the two-mode LSID, we have respectively one and two saddle points, for which the separatrix is three dimensional. Therefore, in the regimes (i)-(iii) the separatrices of $m-1$ saddle points divide the state space in $m$ domains of attraction, each associated with a single attractor. Because trajectories of the particle with different initial conditions do not cross, it is not possible to switch from one region to another without accounting for noise [7].

In the course of a transition from regime (iii) a to (iv) the nonlasing solution becomes unstable. However, as we have seen, the unstable direction is two dimensional instead of one dimensional. It cannot separate the region of the former attractor at $n=0$ in two new regions, each with their own attractor. Importantly, the attractors (saddle points) represented by the solution $n_{\alpha}^{+}\left(n_{\beta}^{+}\right)$and the separatrices do not change significantly, and no new stationary attractors appear. Paradoxically, a particle in the domain of attraction of the former attractor at $n=0$ is not evolving to an attractor anymore, but it also cannot escape to another domain of attraction or to infinity. To resolve this issue, this domain must contain a nonstationary attractor, or limit cycle.

If the frequency of the limit cycle is $\omega_{c}$, one generally expects the emission to occur at a comb of frequencies separated by $\omega_{c}, \omega_{n}=e V / \hbar+n \omega_{c}$. Below we consider the limit of strong coupling where the emission predominantly occurs at two frequencies corresponding to $n= \pm 1$.

\section{B. Perturbative analysis}

In the limit of $G \gg 1$, it is possible to perform a perturbative analysis of the regime (iv). We use the full time dependent Eq. (10). Here, we perform this analysis only up to first order in $G^{-1}$. The results of this subsection explain key features of the numerical results presented in the next subsection.

To analyze Eq. (10) perturbatively, we expand the fields in a series of $G^{-1}: b_{j}=b_{j}^{(0)}+G^{-1} b_{j}^{(1)}$ assuming typical time scales of the order of $G^{-1}$. The lowest order equations read

$$
\dot{\tilde{b}}_{1}^{(0)}+i G e^{-i \pi \Phi / \Phi_{0}} \tilde{b}_{2}^{(0)}=0, \quad \dot{\tilde{b}}_{2}^{(0)}+i G e^{i \pi \Phi / \Phi_{0}} \tilde{b}_{1}^{(0)}=0 .
$$

The solutions can be found straightforwardly as

$$
\tilde{b}_{1}^{(0)}(t)=-i \beta e^{-i \pi \Phi / \Phi_{0}} \sin [G t], \quad \tilde{b}_{2}^{(0)}(t)=\beta \cos [G t],
$$

where we have implicitly chosen an origin in time, $t_{0}$. The complex constant $\beta$ has yet to be determined. It will be fixed by the requirement that the part of $b_{j}$ oscillating with frequency $G$, can be fully contained in the leading order that includes $\tilde{b}_{1}^{(0)}(t)$ and $\tilde{b}_{2}^{(0)}(t)$. The higher order terms in the expansion only oscillate with frequencies that are multiples of $G$. The time average of the total number of photons is $|\beta|^{2}=\tilde{n}_{1}^{(0)}+$ $\tilde{n}_{2}^{(0)}$. This quantity is also proportional to the average current through the device.

We continue with the first-order corrections. To find these, we first take the time derivative of Eq. (10) and then collect all terms that are proportional to $G$. To this end, we keep in mind that each time derivative adds a factor of $G$. We find

$$
\begin{aligned}
& G^{-1}\left[\ddot{\tilde{b}}_{1}^{(1)}+i G e^{-i \pi \Phi / \Phi_{0}} \dot{\tilde{b}}_{2}^{(1)}\right]=-\left[i \tilde{\omega}-2 i\left|\tilde{b}_{1}^{(0)}\right|^{2}+\gamma\right] \dot{\tilde{b}}_{1}^{(0)} \\
& \quad-i\left[1-\left(\tilde{b}_{1}^{(0)}\right)^{2}\right]\left(\dot{\tilde{b}}_{1}^{(0)}\right)^{*} .
\end{aligned}
$$

A second expression exist with $b_{1} \leftrightarrow b_{2}$ and $\Phi \rightarrow-\Phi$. This can be used to eliminate $\dot{\tilde{b}}_{2}^{(1)}$ in Eq. (15). Inserting the expressions for the lowest order terms and rewriting the products of harmonic functions we arrive at

$$
\begin{aligned}
\frac{1}{G}\left[\ddot{\tilde{b}}_{1}^{(1)}+G^{2} \tilde{b}_{1}^{(1)}\right] & =-2 \chi \dot{\tilde{b}}_{1}^{(0)}-\frac{|\beta|^{2}}{2} \beta e^{-i \pi \frac{\Phi}{\Phi_{0}}} G \cos [3 G t], \\
\chi & \equiv i \tilde{\omega}+\gamma-i \frac{3|\beta|^{2}}{4}-i \frac{\beta^{*}}{2 \beta}\left(e^{2 i \pi \Phi / \Phi_{0}}-1\right) .
\end{aligned}
$$

There is a similar equation for $\tilde{b}_{2}$ with the term proportional to $\cos [3 G t]$ replaced by $-i|\beta|^{2} \beta G \sin [3 G t] / 2$. These equations describe a driven harmonic oscillator. Since the term proportional to $\chi$ drives exactly at the resonance frequency $G$, and the frequencies of the higher order terms should only be multiples of $G$, we require $\chi=0$. This sets $\beta$

$$
\begin{gathered}
\left|\beta_{ \pm}\right|^{2}=\frac{4}{3}\left[ \pm \sqrt{\sin ^{2}\left[\pi \Phi / \Phi_{0}\right]-\gamma^{2}}+\tilde{\omega}\right], \\
\gamma \tan \left[2 \phi_{\beta}^{ \pm}-\pi \Phi / \Phi_{0}\right]=\mp \sqrt{\sin ^{2}\left[\pi \Phi / \Phi_{0}\right]-\gamma^{2}},
\end{gathered}
$$

with $\phi_{\beta}^{ \pm}$the phase of $\beta_{ \pm}$. We note that in this limit, $|\beta|^{2}$ and therefore the leading-order term of the average current is independent of the coupling constant $G$. The first-order terms are readily calculated:

$$
\tilde{b}_{1}^{(1)}=\frac{e^{-i \pi \frac{\Phi}{\Phi_{0}}}}{16 G}|\beta|^{2} \beta \cos [3 G t], \quad \tilde{b}_{2}^{(1)}=i \frac{|\beta|^{2} \beta}{16 G} \sin [3 G t] .
$$

These variations have an extra factor of $i$ compared to the leading order, and are therefore perpendicular to it in the complex plane.

The correction to the number, $\delta \tilde{n}_{j}=\tilde{n}_{j}-\tilde{n}_{j}^{(0)}$, is at least of the order $G^{-2}$. The phase between $\tilde{b}_{1}$ and $\tilde{b}_{2}$ is, up to first order, given by $\pi\left(2 \Phi-\Phi_{0}\right) / 2 \Phi_{0}$.

\section{Numerics}

To validate the analytical results of the previous subsection, we have performed a numerical analysis. We study the average current through the two-mode LSID in the limit cycle regime (iv), and the trajectory of the limit cycle.

The analysis is based on the numerical integration of the differential equations in Eq. (10). The initial condition is chosen close to $b_{j}=0$ and the parameters are chosen to achieve the limit cycle regime. To converge to the limit cycle within a reasonable amount of integration time, we choose a sufficiently large damping, $\gamma=0.05$, which is still small enough for all essential features to be as described in the previous section. We integrate the differential equation from $t=0$ up to $t=25 / \gamma$. A time interval of $\delta t=1 / \gamma$ at the end is used to represent the limit cycle, $b_{j}^{l c}(t)$.

The data of $b_{j}^{l c}(t)$ are used to plot several quantities. We use the raw data to demonstrate a few aspects of the limit cycle. The real and imaginary parts of $b_{j}^{l c}(t)$ are plotted in 


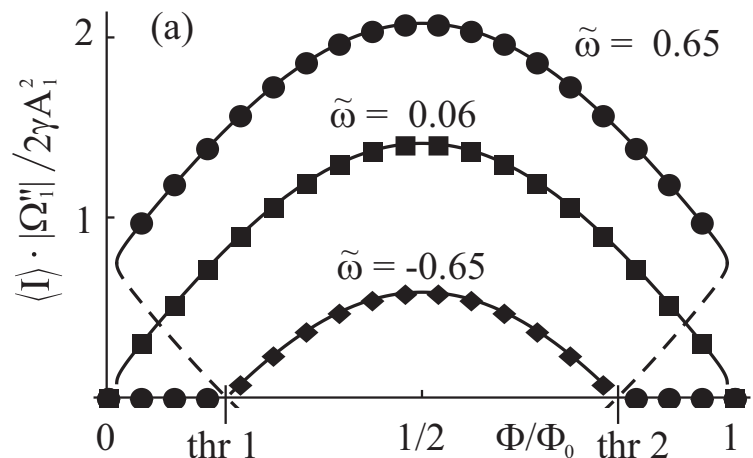

(b)
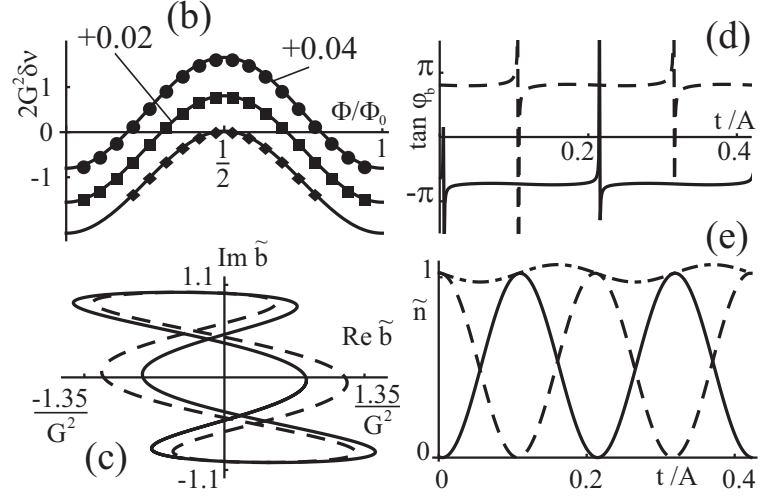

FIG. 5. Limit cycles in regime (iv) of the two-mode LSID, with $G=15$ and $\gamma=0.05$. (a) The average current as a function of flux. The circles, squares, and diamonds correspond to numerical results for three values of the detuning, as indicated in the panel. The solid (dashed) lines are $\left|\beta_{ \pm}\right|^{2}$, corresponding to stable (unstable) limit cycle solutions. The values of the flux labeled with "thr 1" and "thr 2" are lasing thresholds for the solutions with $|\tilde{\omega}|=0.56$. The solution at positive detuning is bistable in the nonlasing regime, with a stable nonlasing state $(I=0)$. This is similar to the earlier discussed regime (iii). Close to $\Phi / \Phi_{0}=0$ and 1 , a regime similar to (i) b exists. (b) The variation of the relative cycle frequency shift $\delta v$ with flux. The circles, squares, and diamonds correspond to the results in (a). The solid lines are fits with the function $\eta_{1} \sin ^{2}\left[\pi \Phi / \Phi_{0}\right]-\eta_{2}$. The coefficients $\left(\eta_{1}, \eta_{2}\right)$ are respectively given by $(0.074,0.064),(0.071,0.067)$, and $(0.068,0.067)$. Two curves are shifted by an amount indicated in the panel. (c) Trajectory of the limit cycle with $\Phi / \Phi_{0}=1 / 4$. The solid (dashed) line corresponds to $\tilde{b}_{1}\left(\tilde{b}_{2}\right)$. The trajectory is rotated over an angle of $0.87 \pi(1.12 \pi)$ to align the long axis of the cycle with the vertical axis of the plot. (d) and (e) The optical phase and number of photons corresponding to the trajectories in the limit cycle of (c). The dash-dotted line in (e) is the sum of the solid and dashed lines.

a parametric plot to show its trajectory, while the modulus and phase of $b_{j}^{l c}(t)$ are plotted as a function of time. The frequency of the limit cycle is shifted from $G$ by $G \delta v \simeq G^{-1}$. We extract the value of $|\beta|^{2}$ by fitting $\left|b_{j}^{l c}(t)\right|^{2}$ to a function of the form $\left|\beta_{j}\right|^{2}\left\{1+\sin \left[2 G(1-\delta v) t+\kappa_{j}\right]\right\}$, corresponding to the leading-order solutions, $n_{1}^{(0)}$ and $n_{2}^{(0)}$. The higher orders are small, being of order $G^{-2}$.

The results of the numerical analysis are shown in Fig. 5. In panel (a), we show the average current through the two-mode LSID as a function of the flux, $\Phi$, for three values of the detuning. We first remark that the expression for $\left|\beta_{+}\right|^{2}$ nicely fits the numerical results. Interestingly, we find two different regimes that remind us of the regimes (ii) and (iii) of the time-independent states. Inevitably, this bistable regime also involves an unstable limit cycle, which we expect to be represented by $\beta_{-}$. With this observation, we conclude that the limit cycle states display parameter dependencies and properties similar to those of the time-independent states investigated in Secs. IV and V. In particular, we find the regimes that are analogous to the regimes (i) b, (ii), and (iii) and the possibility of hysteresis as described in Sec. IV. With this, we review our understanding of the regimes (ii) b, (iii) a (only at positive $\omega$ ), and (iii) b, which were introduced in Sec. V C. We only made notice of the existence of stationary states in these regimes, but we expect that all these regimes also contain a stable and an unstable limit cycle state, represented by $\beta_{ \pm}$.

Panel (b) of Fig. 5 shows the relative shift of the cycle frequency $\delta v=\left(1-\omega_{c} / G\right)$, which is of the order of $G^{-2}$.

In the panels (c)-(e), the raw data are used to show the trajectory of the limit cycle in a parametric plot, and the modulus and phase as a function of time. The trajectory matches the prediction of the previous section. The long axis of the paths correspond to the leading orders $b_{j}^{(0)}$, while the short axis corresponds to the first-order corrections, $b_{j}^{(1)}$. The difference in shape between the trajectories of $\tilde{b}_{1}$ and $\tilde{b}_{2}$ results from the corrections in the perturbation expansion of order $G^{-2}$ and higher. In the panel (e), the moduli $\left|\tilde{b}_{j}(t)\right|^{2}$ are shown separately and as a sum, $\tilde{n}_{1}(t)+\tilde{n}_{2}(t)$, which is proportional to the current. The oscillation amplitude of the current depends on the relative phase of the $b_{j}(t)$.

We have described the limit cycles in the two-mode LSID. The dependence of the limit cycle states on flux and detuning is rather similar to that of the time-independent stationary states. Generally, the emission spectrum in this case consists of a comb of equally separated frequencies $\omega_{n}=e V / \hbar+\omega_{c} n$. Interestingly, in the limit of strong coupling the emission spectrum consists of two discrete frequencies corresponding to $n= \pm 1$. This is therefore a dual-mode lasing state, in contrast to the states in regime (ii) that are single-mode lasing states. The two-mode LSID can thus lase at a single frequency, at $\tilde{\omega} \simeq \pm G$, or at two frequencies at $\tilde{\omega} \lesssim 1$. We stress that the occurrence of the dual-mode lasing regime (iv) is crucially related to the coupling of the superconductors to the resonator modes. Without this coupling, we cannot use the flux to create the instability of the regime (iv), that results in the dual-mode lasing.

\section{CONCLUSIONS}

We summarize the results of the article and sketch some prospectives of HJL-based devices.

We have studied two device setups reminiscent of a superconducting quantum interference device (SQUID), where the regular Josephson junctions are replaced by HJLs: the groups of quantum emitters, emitting in a resonator mode, of which the optically active eigenstates are coupled to both superconducting leads. In the first setup investigated, both groups of quantum emitters emit in a single resonator mode, while in the second setup they emit in two separate resonator modes, which are coupled optically. These setups 
were referred to as, respectively, "single-mode LSID" and "two-mode LSID." In both devices parameter regimes exist that support lasing. The occurrence of nonlasing, lasing, and multistable regimes is equivalent to what is found in a regular HJL. Additionally, the LSIDs also depend on the magnetic flux that threads the superconducting loop of the SQUID. It was found that the LSIDs can operate as a flux-tunable regular single-mode HJL. Indeed, parameter regimes exist where the lasing in the LSIDs can be turned on and off by changing the magnetic flux only. In this context, the occurrence of bistable regimes leads for certain parameter regimes to hysteretic behavior upon performing flux sweeps.

The two-mode LSID has been studied in the weak- and the strong-coupling limits and for a symmetric choice of parameters. In the weak-coupling limit, the device is equivalent to two single HJLs that perturb each other only slightly. A weak dependence on the flux is found. In the strong-coupling limit, the device develops lasing instabilities at detunings of the order of the coupling constant, both positive and negative. At these values of the detuning, the device is similar to a single-mode LSID. Studying the symmetric choice of parameters revealed a new lasing instability in the vicinity of zero detuning, which was investigated in the limit of strong coupling. Here, the device exhibits lasing that is predominantly occurring at two frequencies, which are separated by approximately twice the coupling strength. For such dual-mode lasing, there are regimes similar to the ones of the time-independent states: a nonlasing, a lasing, and a bistable one.

The connection between superconductivity and optics achieved with the HJL devices promises a set of novel applications, this article providing an example thereof. With these prospects, the emerging field of superconducting optoelectronics looks rather promising.

Even more possibilities would emerge for arrays of HJLs. It is easy to extend the design idea of the two-mode LSID to an $n$-mode LSID.

The setup for such an $n$-mode LSID consists of $n$ HJLs in parallel, all sharing the same pair of superconducting electrodes. This guarantees that the devices are driven at the same frequency. Note that there are $n-1$ superconducting loops in this circuit, making it possible to tune the superconducting phase differences of each HJL. An optical coupling between the nearest HJLs is provided. The dynamics is described by a set of $2 n$ equations; those generalize Eq. (10). Each of these equations contains two coupling terms. Linearized equations give $n$ resonant modes. If the detuning matches the resonant frequencies, we expect a single-mode lasing. Otherwise, the lasing regimes may become complex, involving limit cycles and perhaps even chaos. The lasing regimes can be tuned with changing the fluxes in the loops.

To experimentally realize the two-mode LSID and the $n$-mode LSID, a strong constraint is imposed on the spatial dimensions of the optical resonators. These need to be smaller than the SQUID loop, which can be of the order of a micron in diameter [16]. This can be achieved using optical microresonators. For instance, using photonic crystals, one can realize arrays of resonators with nearest-neighbor type couplings, where the resonators are separated by distances of the order of a micron [17].

The $n$-mode LSID is a fairly straightforward extension of the ideas of this article. It is reminiscent to the arrays of Josephson junctions [18] that can be regarded as a realization of artificial solids. Similarly to the Josephson junction arrays, there are rich design possibilities for such HJL devices. One could design any kind of setup with superconducting loops, in 1D, 2D, or even 3D, incorporate as many HJLs as necessary, and couple those optically with each other. The coupling does not even have to be limited to the nearest neighbors. In principle, it can be realized with any number of neighbors, and with varying coupling strengths. This would open up a new field of research, where the physical phenomena typical for Josephson arrays [19-21] merge with optics and lasing.

\section{ACKNOWLEDGMENTS}

We acknowledge financial support from the Dutch Science Foundation NWO/FOM.
[1] S. De Franceschi, L. Kouwenhoven, C. Schönenberger, and W. Wernsdorfer, Nat. Nanotechnol. 5, 703 (2010).

[2] Y. J. Doh, J. A. van Dam, A. L. Roest, E. P. A. M. Bakkers, L. P. Kouwenhoven, and S. De Franceschi, Science 309, 272 (2005).

[3] V. Mourik, K. Zuo, S. M. Frolov, S. R. Plissard, E. P. A. M. Bakkers, and L. P. Kouwenhoven, Science 336, 1003 (2012).

[4] Y. Asano, I. Suemune, H. Takayanagi, and E. Hanamura, Phys. Rev. Lett. 103, 187001 (2009).

[5] M. Khoshnegar and A. H. Majedi, Phys. Rev. B 84, 104504 (2011); F. Hassler, Yu. V. Nazarov, and L. P. Kouwenhoven, Nanotechnology 21, 274004 (2010).

[6] F. Godschalk, F. Hassler, and Yu. V. Nazarov, Phys. Rev. Lett. 107, 073901 (2011).

[7] F. Godschalk and Yu. V. Nazarov, Phys. Rev. B 87, 094511 (2013).

[8] F. Godschalk and Yu. V. Nazarov, Europhys. Lett. 103, 28005 (2013).
[9] R. C. Jaklevic, J. Lambe, A. H. Silver, and J. E. Mercereau, Phys. Rev. Lett. 12, 159 (1964).

[10] M. Tinkham, Introduction to Superconductivity, 2nd edition (McGraw-Hill, New York, 1996).

[11] P. Recher, Yu. V. Nazarov, and L. P. Kouwenhoven, Phys. Rev. Lett. 104, 156802 (2010).

[12] Y. Aharonov and D. J. Bohm, Phys. Rev. 115, 485 (1959).

[13] M. O. Scully and W. E. Lamb, Phys. Rev. 159, 208 (1967).

[14] A. H. Nayfeh and D. T. Mook, Nonlinear Oscillations (Wiley, Weinheim, 2007).

[15] H. A. Haus and W. Huang, Proc. IEEE 79, 1505 (1991).

[16] J.-P. Cleuziou, W. Wernsdorfer, V. Bouchiat, T. Ondarçuhu, and M. Monthioux, Nat. Nanotechnol. 1, 53 (2006).

[17] H. Altug and J. Vučković, Appl. Phys. Lett. 84, 161 (2004).

[18] R. Fazio and H. S. J. van der Zant, Phys. Rep. 355, 235 (2001). 
[19] L. J. Geerligs, M. Peters, L. E. M. de Groot, A. Verbruggen, and J. E. Mooij, Phys. Rev. Lett. 63, 326 (1989).

[20] M. S. Rzchowski, S. P. Benz, M. Tinkham, and C. J. Lobb, Phys. Rev. B 42, 2041 (1990).
[21] V. L. Berezinskii, Sov. Phys. JETP 32, 493 (1971); J. M. Kosterlitz and D. J. Thouless, J. Phys. C 6, 1181 (1973); J. E. Mooij, B. J. van Wees, L. J. Geerligs, M. Peters, R. Fazio, and G. Schön, Phys. Rev. Lett. 65, 645 (1990). 